\title{
Pre-Exposure Prophylaxis (PrEP) for HIV-1 Prevention: The Promise and Challenge
}

\section{Kaio Kitazato}

Department of Molecular, Microbiology and Immunology, Nagasaki University, Nagasaki, Japan

There is still no effective HIV vaccine available, pre-exposure prophylaxis (PrEP) holds promise as a new comprehensive tool for HIV prevention. PrEP is an experimental HIV-prevention strategy using antiretroviral (ARV) agents to protect HIV-negative individuals from HIV infection. Several randomized, blinded, placebo-controlled trials of oral or topical antiretroviral compounds have confirmed that ARV-based PrEP can significantly lower the risk of sexual acquisition of HIV [1]. After a six-year, nine-country clinical trial, the US NIH has announced that early detection and treatment of HIV could cut transmission of HIV by up to 96 per cent [2]. These results suggest that well-designed clinical trials of PrEP have the potential to control the epidemic until a safe and effective HIV vaccine is available.

However, the appropriate clinical use of PrEP faces many challenges. PrEP efficacy is highly dependent on medication adherence.
Nonadherence seems to be the main reason for the PrEP failures observed in previous trials [3]. The continued drug exposure maintains the selection pressure eliciting mutations allowing the rapid emergence of drug-resistant HIV-1 strains. The appearance of cross-resistance and also cost severely limit the long-term treatment and use of PrEP for HIV prevention. How we use the limited ARV resources, for treatment, for prevention, or for both, also is a future challenge. We therefore need to develop more low-cost, effective ARVs for PrEP HIV prevention.

\section{References}

1. http://www.cdc.gov/hiv/prep/

2. http://www.nih.gov/news/health/may2011/niaid-12.htm

3. Hurt C B, Eron JJ Jr, Cohen MS (2011) Pre-Exposure Prophylaxis and Antiretroviral Resistance: HIV Prevention at a Cost? Clin Infect Dis [Epub
*Corresponding author: Kaio Kitazato, Ph.D, Department of Molecular Microbiology and Immunology, Graduate School of Biomedical Sciences, Nagasaki University, Nagasaki City, 1-14 Bunkyo-machi, Nagasaki, 8528521, Japan, Tel:+81-95-8192457; Fax: +81-95-819-2898; E-mail: kitazato8@gmail.com

Received November 11, 2011; Accepted November 14, 2011; Published November 20, 2011

Citation: Kitazato K (2011) Pre-Exposure Prophylaxis (PrEP) for HIV-1 Prevention The Promise and Challenge. J AIDS Clinic Res 2:101e. doi:10.4172/2155$6113.1000101 \mathrm{e}$

Copyright: @ 2011 Kitazato K. This is an open-access article distributed under the terms of the Creative Commons Attribution License, which permits unrestricted use, distribution, and reproduction in any medium, provided the original author and source are credited. 